\title{
Splines of Complex Order: Fourier, Filters and Fractional Derivatives
}

\author{
Brigitte Forster \\ Zentrum Mathematik, M6, Technische Universtität München \\ Boltzmannstr. 3, 85747 Garching, Germany \\ forster@ma.tum.de \\ Peter Massopust \\ Institute of Biomathematics and Biometry, Helmholtz Zentrum München \\ Ingolstädter Landstr. 1, 85764 Neuherberg, Germany \\ peter.massopust@helmholtz-muenchen.de \\ and \\ Zentrum Mathematik, M6, Technische Universtität München \\ Boltzmannstr. 3, 85747 Garching, Germany \\ massopust@ma.tum.de
}

\begin{abstract}
For the Schoenberg (polynomial) B-splines, interesting relations between their functional representation, Dirichlet averages, and difference operators are known. We use these relations to extend B-splines to an arbitrary (infinite) sequence of knots and to higher dimensions. The Fourier domain representation of multidimensional complex B-splines, their twoscale relation and an explicit time domain representation are given for the case of equidistantly distributed knots on rays. In addition, we show that complex B-splines are solutions of the sampling/interpolation problem

$$
\mathcal{D}^{z} f=\sum_{k=0}^{\infty} a_{k} \delta(\bullet-k)
$$

of complex order $z, \operatorname{Re} z>1$.

Key words and phrases: Complex B-splines, Dirichlet means, Scaling relation, Ridge functions, Multivariate splines, Fractional derivative and integral, Sampling series, Dirac comb, Mittag-Leffler function, Lizorkin space
\end{abstract}

2010 AMS Mathematics Subject Classification - 41A15, 26A33,41A63

\section{Complex B-Splines}

Complex B-splines are a natural extension of the classical Curry-Schoenberg (polynomial) B-splines [1] and the fractional splines first investigated in [29]. 
The complex B-splines $B_{z}: \mathbb{R} \rightarrow \mathbb{C}$ are defined in the Fourier domain by

$$
\mathcal{F}\left(B_{z}\right)(\omega):=\int_{\mathbb{R}} B_{z}(t) e^{-i \omega t} d t:=\left(\frac{1-e^{-i \omega}}{i \omega}\right)^{z},
$$

for $\operatorname{Re} z>1$. They are well-defined: Since $\left\{\frac{1-e^{-i \omega}}{i \omega} \mid \omega \in \mathbb{R}\right\} \cap\{y \in \mathbb{R} \mid y<0\}=\emptyset$ they reside on the main branch of the complex logarithm. Complex B-splines are elements of $L^{1}(\mathbb{R}) \cap L^{2}(\mathbb{R})$ and they have several interesting basic properties, which are discussed in [3]. In the following, we summarize the most important ones for our purposes.

To this end, let $\operatorname{Re} z, \operatorname{Re} z_{1}, \operatorname{Re} z_{2}>1$. Complex B-splines $B_{z}$ are piecewise polynomials of complex degree. Due to their decay in the frequency domain that is induced by the polynomial $\omega^{z}$ in the denominator of (1), complex B-splines $B_{z}$ are elements of the Sobolev space $W_{2}^{r}(\mathbb{R})$ for $r<\operatorname{Re} z-\frac{1}{2}$ (with respect to the $L^{2}$-norm and with weight $\left.\left(1+|x|^{2}\right)^{r}\right)$. The smoothness of their Fourier transform yields fast decay in the time domain:

$$
B_{z}(x) \in \mathcal{O}\left(x^{-m}\right), \quad \text { for } m<\operatorname{Re} z+1 \text {, as }|x| \rightarrow \infty .
$$

Complex B-splines satisfy the recursion formula $B_{z_{1}} * B_{z_{2}}=B_{z_{1}+z_{2}}$, are scaling functions, and generate multiresolution analyses and wavelets. But in general, they do not have compact support. Furthermore, they relate difference and differential operators.

The aims for this paper are twofold. Firstly, we take a closer look at a multivariate extension of complex B-splines, in particular, their explicit time domain and frequency domain extensions. Secondly, we show the relation of splines of complex order to fractional differential operators and to sampling series.

We proceed as follows: In Section 2 we establish the basic theory of B-splines and present their relations to Dirichlet averages and difference operators. In Section 3 we introduce the extension to multivariate splines. Sections 4 and 5 are concerned with explicit frequency and time domain representations of these multivariate splines and their properties. Finally, in Section 6 we present the relation between splines of complex order and complex differential operators and weighted Dirac combs.

\section{From univariate to multivariate splines}

The relations between Dirichlet averages and splines was first established by Carlson [4, 5] and since then has attracted many authors, e.g. [6, 14, 22, 32]. In this article, we extend the univariate splines to a multivariate setting by exploiting their relations to difference operators and Dirichlet averages. For a schematic sketch, see Figure 1. 


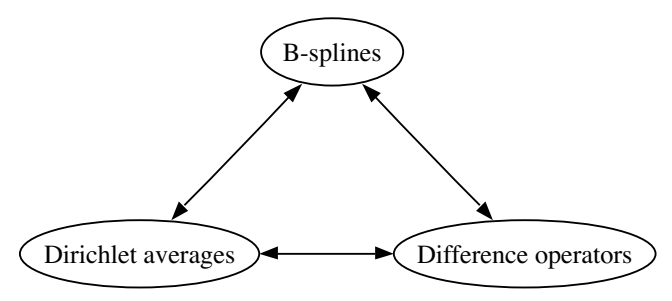

Figure 1: Relations between classical B-splines, difference operators, and Dirichlet averages

\subsection{Time-domain representation of univariate complex B-splines}

In (1), we have defined univariate complex B-splines in the frequency domain. The Fourier inversion shows that these functions are piecewise polynomials of complex degree:

Proposition 1. [3] Complex B-splines have a time-domain representation of the form

$$
B_{z}(t)=\frac{1}{\Gamma(z)} \sum_{k \geq 0}(-1)^{k}\left(\begin{array}{l}
z \\
k
\end{array}\right)(t-k)_{+}^{z-1},
$$

where the equality holds point-wise for all $t \in \mathbb{R}$ and in the $L^{2}(\mathbb{R})$-norm. Here,

$$
\left(\begin{array}{l}
z \\
k
\end{array}\right):=\frac{\Gamma(z+1)}{\Gamma(k+1) \Gamma(z-k+1)},
$$

and

$$
t_{+}^{z}=\left\{\begin{array}{cl}
t^{z}=e^{z \ln t}, & \text { if } t>0 \\
0, & \text { if } t \leq 0,
\end{array}\right.
$$

is the truncated power function, and $\Gamma: \mathbb{C} \backslash \mathbb{Z}_{0}^{-} \rightarrow \mathbb{C}$ denotes the Euler Gamma function.

In comparison, the (polynomial) cardinal B-spline $B_{n}, n \in \mathbb{N}$, has the similar representation

$$
\begin{aligned}
B_{n}(t) & =\frac{1}{(n-1) !} \sum_{k=0}^{n}(-1)^{k}\left(\begin{array}{l}
n \\
k
\end{array}\right)(t-k)_{+}^{n-1} \\
& =\frac{1}{\Gamma(n)} \sum_{k=0}^{\infty}(-1)^{k}\left(\begin{array}{l}
n \\
k
\end{array}\right)(t-k)_{+}^{n-1}
\end{aligned}
$$

\subsection{Relation to difference operators}

It is well-known that in the construction of the Curry-Schoenberg B-splines difference operators are deeply involved. The same is true for complex B-splines. 
To establish the corresponding relation, let us first recall the definition of the backward difference operator $\nabla$.

Let $g: \mathbb{R} \rightarrow \mathbb{C}$ be a function. Then the backward difference operator $\nabla=\nabla^{1}$ is recursively defined as follows:

$$
\begin{aligned}
\nabla g(t) & =g(t)-g(t-1) \\
\nabla^{n+1} g(t) & =\nabla\left(\nabla^{n} g(t)\right), \quad \text { for } n \in \mathbb{N}
\end{aligned}
$$

This definition yields the explicit representation

$$
\nabla^{n} g(t)=\sum_{k=0}^{n}\left(\begin{array}{l}
n \\
k
\end{array}\right)(-1)^{k} g(t-k) .
$$

For the cardinal B-splines $B_{n}$ we can write:

$$
\begin{aligned}
B_{n}(t) & =\frac{1}{(n-1) !} \sum_{k=0}^{n}(-1)^{k}\left(\begin{array}{l}
n \\
k
\end{array}\right)(t-k)_{+}^{n-1} \\
& =\frac{1}{(n-1) !} \nabla^{n} t_{+}^{n-1} .
\end{aligned}
$$

This invites us to define a complex difference operator based on the representation (2) of the form stated below:

Definition 2. $[3,9]$ The difference operator $\nabla^{z}$ of complex order $z$ is defined as

$$
\nabla^{z} g(t):=\sum_{k=0}^{\infty}(-1)^{k}\left(\begin{array}{l}
z \\
k
\end{array}\right) g(t-k), \quad z \in \mathbb{C}, \quad \operatorname{Re} z \geq 1
$$

Hence a second time domain representation of the complex B-spline is given by

$$
B_{z}(t)=\frac{1}{\Gamma(z)} \nabla^{z} t_{+}^{z-1}
$$

In a similar way, we can establish a relation to divided differences. Recall that for a knot sequences $\left\{t_{0}, \ldots, t_{n}\right\} \subset \mathbb{R}, n \geq 1$, divided differences are recursively defined as follows. Let $g: \mathbb{R} \rightarrow \mathbb{C}$ be some function.

$$
\begin{aligned}
{\left[t_{0}\right] g } & =g\left(t_{0}\right), \\
{\left[t_{0}, \ldots, t_{n}\right] g } & =\frac{\left[t_{0}, \ldots, t_{n-1}\right] g-\left[t_{1}, \ldots, t_{n}\right] g}{t_{0}-t_{n}} \\
& =\sum_{j=0}^{n} \frac{g\left(t_{j}\right)}{\prod_{l \neq j}\left(t_{j}-t_{l}\right)} .
\end{aligned}
$$


For the cardinal B-spline,

$$
\begin{aligned}
B_{n}(t) & =\frac{1}{(n-1) !} \sum_{k=0}^{n}(-1)^{k}\left(\begin{array}{l}
n \\
k
\end{array}\right)(t-k)_{+}^{n-1} \\
& =n \sum_{k=0}^{n}(-1)^{k} \frac{1}{k !(n-k) !}(t-k)_{+}^{n-1} \\
& =(-1)^{n} n \sum_{k=0}^{n} \frac{(t-k)_{+}^{n-1}}{\prod_{l \neq k}(k-l)} \\
& =(-1)^{n} n[0,1, \ldots, n](t-\bullet)_{+}^{n-1}
\end{aligned}
$$

(The factor $(-1)^{n}$ is due to our representation of the cardinal B-spline via backward difference operators.)

The same ideas give rise to the definition of complex divided differences.

Definition 3. Let $g: \mathbb{R} \rightarrow \mathbb{C}$ be a function. We define the complex divided differences for the knot sequence $\mathbb{N}_{0}:=\mathbb{N} \cup\{0\}$ via

$$
\left[z ; \mathbb{N}_{0}\right] g:=\sum_{k \geq 0}(-1)^{k} \frac{g(k)}{\Gamma(z-k+1) \Gamma(k+1)} .
$$

Then the complex B-spline can be written as

$$
B_{z}(t)=z\left[z, \mathbb{N}_{0}\right](t-\bullet)_{+}^{z-1} .
$$

Comparing "old" and "new" divided difference operators for $z=n \in \mathbb{N}$, yields

$$
(-1)^{n}[0,1, \ldots n]=\left[n, \mathbb{N}_{0}\right]
$$

Proposition 4. $[10,9]$ Let $\operatorname{Re} z>0$ and $g \in \mathcal{S}\left(\mathbb{R}^{+}\right)$. Then

$$
\left[z ; \mathbb{N}_{0}\right] g=\frac{1}{\Gamma(z+1)} \int_{\mathbb{R}} B_{z}(t) g^{(z)}(t) d t
$$

where $g^{(z)}=W^{z} g$ is the complex Weyl derivative:

For $n=\lceil\operatorname{Re} z\rceil, \nu=n-z$,

$$
W^{z} g(t):=(-1)^{n} \frac{d^{n}}{d t^{n}}\left[\frac{1}{\Gamma(\nu)} \int_{t}^{\infty}(x-t)^{\nu-1} g(x) d x\right] .
$$

\section{Sketch of proof:}

$$
\begin{aligned}
\frac{1}{\Gamma(z+1)} \int_{\mathbb{R}} B_{z}(t) g^{(z)}(t) d t & =\frac{1}{\Gamma(z+1)} \int_{\mathbb{R}} z\left[z, \mathbb{N}_{0}\right](t-\bullet)_{+}^{z-1} W^{z} g(t) d t \\
& =\left[z, \mathbb{N}_{0}\right] \frac{1}{\Gamma(z)} \int_{\bullet}^{\infty}(t-\bullet)_{+}^{z-1} W^{z} g(t) d t \\
& =\left[z, \mathbb{N}_{0}\right] W^{-z} W^{z} g=\left[z, \mathbb{N}_{0}\right] g .
\end{aligned}
$$


Here, $W^{-z} f=\frac{1}{\Gamma(z)} \int_{\bullet}^{\infty}(t-\bullet)_{+}^{z-1} f(t) d t$ is the complex Weyl integral of the function $f$, i.e., the inverse operator of $W^{z}$.

Now we are able to establish a first relation between divided difference operators and Dirichlet averages.

Proposition 5. (Generalized Hermite-Genocchi-Formula: Divided Differences and Dirichlet Averages) [10,9]

Let $\Delta^{\infty}$ be the infinite-dimensional simplex

$$
\Delta^{\infty}:=\left\{u:=\left(u_{j}\right) \in\left(\mathbb{R}_{0}^{+}\right)^{\mathbb{N}_{0}} \mid \sum_{j=0}^{\infty} u_{j}=1\right\}=\underset{\longleftarrow}{\lim } \Delta^{n},
$$

defined as the projective limit of the finite dimensional simplices $\Delta^{n}$, and let $\mu_{e}^{\infty}$ be the generalized Dirichlet measure defined by the projective limit

$$
\mu_{e}^{\infty}=\lim _{\longleftarrow} \Gamma(n+1) \lambda^{n}
$$

where $\lambda^{n}$ denotes Lebesgue measure on $\Delta^{n}$. Then

$$
\begin{aligned}
{\left[z, \mathbb{N}_{0}\right] g } & =\frac{1}{\Gamma(z+1)} \int_{\Delta \infty} g^{(z)}\left(\mathbb{N}_{0} \cdot u\right) d \mu_{e}^{\infty}(u) \\
& =\frac{1}{\Gamma(z+1)} \int_{\mathbb{R}} B_{z}(t) g^{(z)}(t) d t
\end{aligned}
$$

for all real-analytic $g \in \mathcal{S}\left(\mathbb{R}^{+}\right)$.

\subsection{Splines and Dirichlet averages}

Up to now we have considered complex B-splines with knot sequence $\mathbb{N}_{0}$ and derived new difference operators and established a relationship to Dirichlet averages, as indicated in the diagram in Fig. 1:

$$
\text { B-splines } \rightarrow \text { Difference operators } \rightarrow \text { Dirichlet averages. }
$$

Our next step consists of generalizing the present setting to include weights in the Dirichlet measures by traveling through the diagram in another way: From Dirichlet averages with more general knot sequences $\tau$ and with weights $\rightarrow$ Generalized B-splines with knot sequence $\tau \rightarrow$ Difference operators.

Let $b \in \mathbb{R}_{+}^{\infty}$ be a weight vector and $\tau=\left\{t_{k}\right\}_{k \in \mathbb{N}_{0}} \in \mathbb{R}_{+}^{\mathbb{N}_{0}}$ an increasing sequence of knots with $\lim \sup _{k \rightarrow \infty} \sqrt[k]{t_{k}} \leq \rho<e$, some $\rho>0$.

Definition 6. A complex B-spline $B_{z}(\bullet \mid b ; \tau)$ with weight vector $b$ and knot sequence $\tau$ is a function satisfying

$$
\int_{\mathbb{R}} B_{z}(t \mid b ; \tau) g^{(z)}(t) d t=\int_{\Delta^{\infty}} g^{(z)}(\tau \cdot u) d \mu_{b}^{\infty}(u)
$$


for all real-analytic $g \in \mathcal{S}\left(\mathbb{R}^{+}\right)$. Here, $\mu_{b}^{\infty}=\lim _{\longleftarrow} \mu_{b}^{n}$ is the projective limit of Dirichlet measures with densities

$$
\frac{\Gamma\left(b_{0}\right) \ldots \Gamma\left(b_{n}\right)}{\Gamma\left(b_{0}+\ldots+b_{n}\right)} u_{0}^{b_{0}-1} u_{1}^{b_{1}-1} \ldots u_{n}^{b_{n}-1} .
$$

Since both $W^{z}$ and $W^{-z}$ are linear operators mapping $\mathcal{S}\left(\mathbb{R}^{+}\right)$into itself $[20,26]$ and since the real-analytic functions in $\mathcal{S}\left(\mathbb{R}^{+}\right)$are dense in $\mathcal{S}\left(\mathbb{R}^{+}\right)[23]$, (3) holds for all $g \in \mathcal{S}\left(\mathbb{R}^{+}\right)$. Moreover, since $\mathcal{S}\left(\mathbb{R}^{+}\right)$is dense in $L^{2}\left(\mathbb{R}^{+}\right)$, we deduce that $B_{z}(\bullet \mid b, \tau) \in L^{2}\left(\mathbb{R}^{+}\right)$.

Equation (3) means that we defined a weighted version of a complex B-spline in a weak sense via Dirichlet averages. Referring again to the diagram in Fig. 1, we now move from the generalized B-splines to generalized divided differences.

Definition 7. For knot sequences $\tau \in \mathbb{R}_{+}^{\mathbb{N}_{0}}$ and weight vectors $b \in \mathbb{R}_{+}^{\infty}$ as above, we define the generalized complex divided differences $[z ; b, \tau]$ as follows. Let $g: \mathbb{R} \rightarrow \mathbb{C}$ be some function. Then

$$
[z ; b, \tau] g:=\frac{1}{\Gamma(z)} \int_{\mathbb{R}} B_{z}(t \mid b ; \tau) g^{(z)}(t) d t
$$

for all $g \in \mathcal{S}(\mathbb{R})$. In case $b=e=(1,1,1, \ldots)$ and $\tau=\mathbb{N}_{0}$, we write $[z ; e, \tau]=$ $\left[z ; \mathbb{N}_{0}\right]$.

This definition is compatible with the usual Dirichlet splines. In fact, for all finite $\tau=\tau(n) \in \mathbb{R}_{+}^{n+1}$ and $b=b(n) \in \mathbb{R}_{+}^{n+1}$, and for $z=n \in \mathbb{N}_{0}$ the Dirichlet spline $D_{n}(\bullet \mid b ; \tau)$ of order $n$ is defined by

$$
\begin{aligned}
\int_{\mathbb{R}} g^{(n)}(t) D_{n}(t \mid b ; \tau) d t & =\int_{\Delta^{n}} g^{(n)}(\tau \cdot u) d \mu_{b}^{n}(u) \\
& =: \quad G^{(n)}(b ; \tau)
\end{aligned}
$$

for all $g \in C^{n}(\mathbb{R})$. Here, $G^{(n)}$ is the derivative of $G$, the Dirichlet average of $g$ (see [4], p. 79):

$$
G(b ; \tau)=\int_{\Delta^{n}} g(\tau \cdot u) d \mu_{b}^{n}(u)
$$

\section{Multivariate Complex B-Splines}

To extend complex B-splines to a multivariate setting, we define multivariate B-splines on the basis of ridge functions. Then, we move again through the diagram in Fig. 1: Multivariate B-splines $\rightarrow$ Multivariate difference operators. Results on Dirichlet averages also yield recurrence relations for multivariate Bsplines: Dirichlet averages $\rightarrow$ B-splines. For this step through the diagram we refer to $[18,16]$. 
Note that the approach via ridge functions had already let to an extension of the Curry-Schoenberg-splines to a multivariate setting, e.g. [6, 2, 19, 22]. However, some of these approaches have certain restrictions on the knots and none of them considers splines of complex order.

Given $\boldsymbol{\lambda} \in \mathbb{R}^{s} \backslash\{\mathbf{0}\}$, a direction, and $g: \mathbb{R} \rightarrow \mathbb{C}$ a function. The ridge function $g_{\boldsymbol{\lambda}}$ corresponding to $g$ is defined via

$$
g_{\boldsymbol{\lambda}}: \mathbb{R}^{s} \rightarrow \mathbb{C}, \quad g_{\boldsymbol{\lambda}}(\boldsymbol{x}):=g(\langle\boldsymbol{\lambda}, \boldsymbol{x}\rangle), \quad \text { for all } \boldsymbol{x} \in \mathbb{R}^{s} .
$$

Definition 8. [18] Let $\boldsymbol{\tau}=\left\{\boldsymbol{\tau}^{n}\right\}_{n \in \mathbb{N}_{0}} \in\left(\mathbb{R}^{s}\right)^{\mathbb{N}_{0}}$ a sequence of knots in $\mathbb{R}^{s}$ with $\limsup _{n \rightarrow \infty} \sqrt[n]{\left\|\boldsymbol{\tau}^{n}\right\|} \leq \rho<e$, some $\rho>0$. The multivariate complex B-spline $\boldsymbol{B}_{z}(\bullet \mid b ; \boldsymbol{\tau})$ with weights $b \in \mathbb{R}_{+}^{\mathbb{N}_{0}}$ and knots $\boldsymbol{\tau}$ is defined on ridge functions via

$$
\int_{\mathbb{R}^{s}} g(\langle\boldsymbol{\lambda}, \boldsymbol{x}\rangle) \boldsymbol{B}_{z}(\boldsymbol{x} \mid b ; \boldsymbol{\tau}) d \boldsymbol{x}=\int_{\mathbb{R}} g(t) B_{z}(t \mid b ; \boldsymbol{\lambda} \boldsymbol{\tau}) d t
$$

where $g \in \mathcal{S}\left(\mathbb{R}^{+}\right)$and $\boldsymbol{\lambda} \in \mathbb{R}^{s} \backslash\{\mathbf{0}\}$, such that $\boldsymbol{\lambda} \boldsymbol{\tau}=\left\{\left\langle\boldsymbol{\lambda}, \boldsymbol{\tau}^{n}\right\rangle\right\}_{n \in \mathbb{N}_{0}}$ is separated, i.e., there exists a $\delta>0$, so that $\inf \left\{\left|\left\langle\boldsymbol{\lambda}, \boldsymbol{\tau}^{n}\right\rangle-\left\langle\boldsymbol{\lambda}, \boldsymbol{\tau}^{m}\right\rangle\right|: m, n \in \mathbb{N}_{0}\right\} \geq \delta$.

Since ridge functions are dense in $L^{2}\left(\mathbb{R}^{s}\right)[24]$, we conclude that $\boldsymbol{B}_{z}(\bullet \mid b ; \boldsymbol{\tau}) \in$ $L^{2}\left(\left(\mathbb{R}^{+}\right)^{s}\right)$.

Example 9. (Divided differences in the multivariate case)

Given $b=e:=(1,1,1, \ldots)$. Then for all $g \in \mathcal{S}\left(\mathbb{R}^{\infty}\right)$ :

$$
\begin{aligned}
{[z ; e, \boldsymbol{\tau}] g_{\boldsymbol{\lambda}} } & =[z ; \boldsymbol{\tau}] g_{\boldsymbol{\lambda}}=[z ; \boldsymbol{\tau}] g(\langle\boldsymbol{\lambda}, \bullet\rangle) \\
& =\frac{1}{\Gamma(z)} \int_{\mathbb{R}^{s}} g^{(z)}(\langle\boldsymbol{\lambda}, \boldsymbol{x}\rangle) \boldsymbol{B}_{z}(\boldsymbol{x} \mid e ; \boldsymbol{\tau}) d \boldsymbol{x} \\
& =\frac{1}{\Gamma(z)} \int_{\mathbb{R}} g^{(z)}(t) B_{z}(t \mid e ; \boldsymbol{\lambda} \boldsymbol{\tau}) d t=[z ; \boldsymbol{\lambda} \boldsymbol{\tau}] g
\end{aligned}
$$

for all $\boldsymbol{\lambda} \in \mathbb{R}^{s} \backslash\{\mathbf{0}\}$ such that $\boldsymbol{\lambda} \boldsymbol{\tau}$ is separated.

Example 10. (Multivariate cardinal B-splines) For $n \in \mathbb{N}$ and a finite sequence of knots $\boldsymbol{\tau}=\left\{\boldsymbol{\tau}^{0}, \boldsymbol{\tau}^{1}, \ldots, \boldsymbol{\tau}^{n}\right\}$ :

$$
\begin{aligned}
& {\left[\boldsymbol{\tau}^{0}, \ldots, \boldsymbol{\tau}^{n}\right] g_{\boldsymbol{\lambda}}:=[n ; \boldsymbol{\tau}] g(\langle\boldsymbol{\lambda}, \bullet\rangle) } \\
= & \frac{1}{n !} \int_{\mathbb{R}^{s}} g^{(n)}(\langle\boldsymbol{\lambda}, \boldsymbol{x}\rangle) \boldsymbol{B}_{n}(\boldsymbol{x} \mid e ; \boldsymbol{\tau}) d \boldsymbol{x} \\
= & \frac{1}{n !} \int_{\mathbb{R}} g^{(n)}(t) B_{n}(t \mid e ; \boldsymbol{\lambda} \boldsymbol{\tau}) d t \\
= & {[n ; \boldsymbol{\lambda} \boldsymbol{\tau}] g=\sum_{j=0}^{n} \frac{g\left(\left\langle\boldsymbol{\lambda}, \boldsymbol{\tau}^{j}\right\rangle\right)}{\prod_{l \neq j}\left\langle\boldsymbol{\lambda}, \boldsymbol{\tau}^{j}-\boldsymbol{\tau}^{l}\right\rangle} . }
\end{aligned}
$$


MULTIVARIATE COMPLEX B-SPLINES

\section{Fourier representation of multivariate complex B- splines}

We saw above that both the univariate and the multivariate complex B-splines are $L^{2}$-functions: $B_{z}(\bullet \mid b ; \tau) \in L^{2}(\mathbb{R})$ and $\boldsymbol{B}_{z}(\bullet \mid b ; \boldsymbol{\tau}) \in L^{2}\left(\mathbb{R}^{s}\right)$. Therefore, we can apply the Plancherel transform to both functions and consider their frequency spectrum.

Let $\boldsymbol{\omega}=\left(\omega_{1}, \ldots, \omega_{s}\right) \in \mathbb{R}^{s}$ and let $\boldsymbol{\lambda} \in \mathbb{R}^{s},\|\boldsymbol{\lambda}\|=1$, be the direction of $\boldsymbol{\omega}$, i.e., $\boldsymbol{\omega}=\omega \boldsymbol{\lambda}$ for some $\omega \geq 0$. For $\boldsymbol{x}=\left(x_{1}, \ldots, x_{s}\right) \in \mathbb{R}^{s}$, we obtain as the Fourier transform of the generalized complex B-spline the following expression:

$$
\begin{aligned}
\widehat{B}_{z} & (\omega \mid b ; \boldsymbol{\lambda} \boldsymbol{\tau})= \\
& =\int_{\mathbb{R}^{2}} e^{-i \omega t} B_{z}(t \mid b ; \boldsymbol{\lambda} \boldsymbol{\tau}) d t \\
& =\int_{\mathbb{R}^{s}} e^{-i \omega\langle\boldsymbol{\lambda}, \boldsymbol{x}\rangle} \boldsymbol{B}_{z}(\boldsymbol{x} \mid b ; \boldsymbol{\tau}) d \boldsymbol{x} \\
& =\int_{\mathbb{R}^{s}} e^{-i\langle\boldsymbol{\omega}, \boldsymbol{x}\rangle} \boldsymbol{B}_{z}(\boldsymbol{x} \mid b ; \boldsymbol{\tau}) d \boldsymbol{x} \\
& =\widehat{\boldsymbol{B}}_{z}(\boldsymbol{\omega} \mid b ; \boldsymbol{\tau})=\widehat{\boldsymbol{B}}_{z}(\omega \boldsymbol{\lambda} \mid b ; \boldsymbol{\tau}) .
\end{aligned}
$$

This shows that the frequency spectrum of the multivariate complex B-spline along directions $\boldsymbol{\lambda}$ is given by the spectrum of the univariate spline with knots projected onto these $\boldsymbol{\lambda}$. Note that on the left-hand side, the Fourier transform is one-dimensional, but $s$-dimensional on the right-hand side. I.e., the formula is related to the Central Slice theorem for the Radon transfom [8, 21].

We now assume that the knots are equidistantly distributed on a ray in $\mathbb{R}^{s}$, i.e., $\boldsymbol{\tau}=\boldsymbol{d} \mathbb{N}_{0}$ for some distance vector $\boldsymbol{d} \in \mathbb{R}^{s}$. Then we have

$$
\widehat{\boldsymbol{B}}_{z}(\omega \boldsymbol{\lambda} \mid b ; \boldsymbol{\tau})=\widehat{\boldsymbol{B}}_{z}\left(\omega \boldsymbol{\lambda} \mid b, \boldsymbol{d} \mathbb{N}_{0}\right)=\widehat{\boldsymbol{B}}_{z}\left(\omega \mid b,\langle\boldsymbol{\lambda}, \boldsymbol{d}\rangle \mathbb{N}_{0}\right),
$$

and concretely for weights $b=e=(1,1,1, \ldots)$

$$
\widehat{\boldsymbol{B}}_{z}\left(\omega \boldsymbol{\lambda} \mid e ; \boldsymbol{d} \mathbb{N}_{0}\right)=\left(\frac{1-e^{-i\langle\boldsymbol{\lambda}, \boldsymbol{d}\rangle \omega}}{i \omega}\right)^{z}, \quad \text { in } L^{2}\left(\mathbb{R}^{s}\right) .
$$

For a plot of these functions in 2D, see Figure 2. The images show a sinc-like behavior of $\widehat{\boldsymbol{B}}_{z}\left(\omega \boldsymbol{\lambda} \mid e ; \boldsymbol{d} \mathbb{N}_{0}\right)$ in the direction orthogonal to $\boldsymbol{d}$, and a smooth lowpass behavior in direction of $\boldsymbol{d}$. In fact,

$$
\widehat{\boldsymbol{B}}_{z}\left(\omega \boldsymbol{\lambda} \mid e ; \boldsymbol{d} \mathbb{N}_{0}\right)=0 \quad \Longleftrightarrow \omega\langle\boldsymbol{\lambda}, \boldsymbol{d}\rangle=2 \pi K, \quad K \in \mathbb{Z},
$$

i.e., $\widehat{\boldsymbol{B}}_{z}\left(\omega \boldsymbol{\lambda} \mid e ; \boldsymbol{d} \mathbb{N}_{0}\right)$ vanishes on a line through the origin orthogonal to $\boldsymbol{d}$ and on all parallel lines a distance $2 \pi$ apart:

$$
\omega_{1} d_{1}+\omega_{2} d_{2}=2 \pi K, \quad K \in \mathbb{Z},
$$




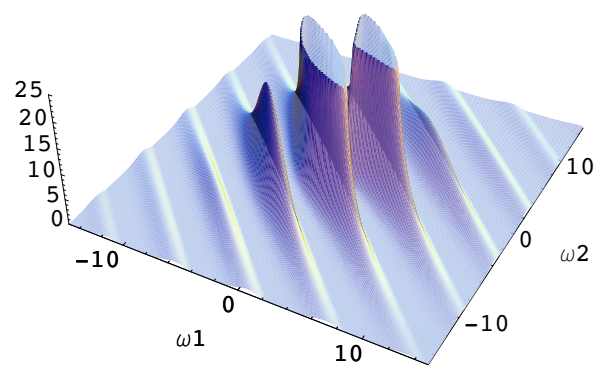

(a)

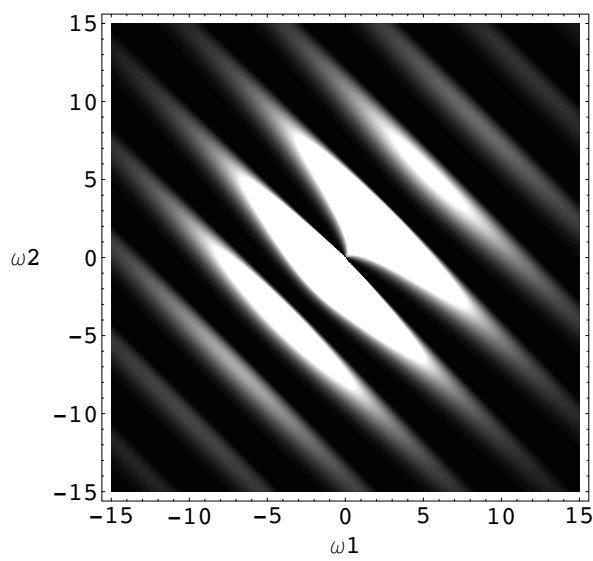

(b)

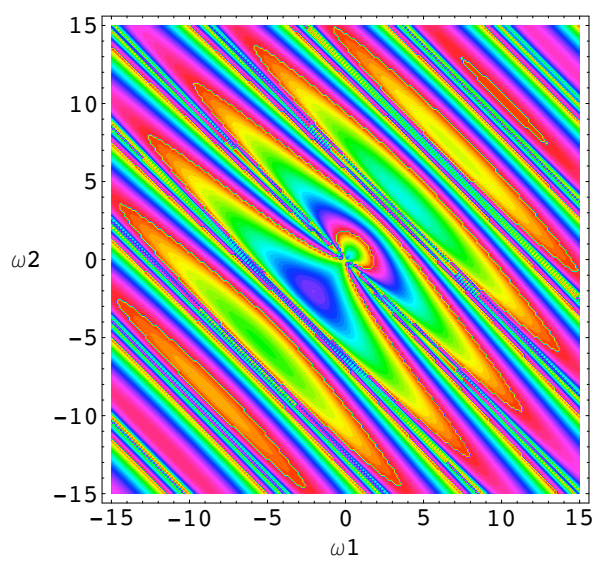

(c)

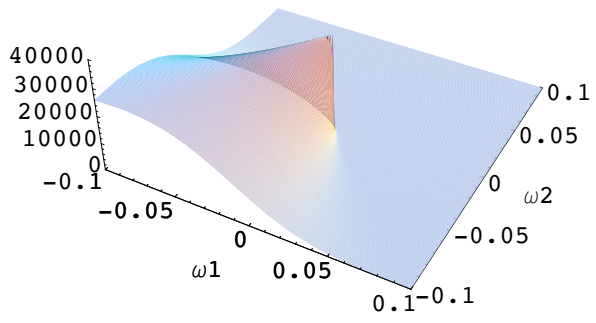

(d)

Figure 2: $\widehat{\boldsymbol{B}}_{z}\left(\boldsymbol{\omega} \mid e, \boldsymbol{d} \mathbb{N}_{0}\right)$, the bivariate complex B-spline in frequency domain. The parameters are $z=3+3 i$ and $\boldsymbol{d}=(1,1)$. (a) Absolute value of the multivariate complex B-spline, (large values are truncated) (b) the corresponding top view (white $=$ large absolute values, black $=0$ ), and (c) the phase. (d) Untruncated modulus of $\widehat{\boldsymbol{B}}_{z}\left(\boldsymbol{\omega} \mid e, \boldsymbol{d} \mathbb{N}_{0}\right)$ in a neighborhood of the origin. Because of the radial construction via ridge functions, the function is discontinuous at the origin. 
where $\boldsymbol{\omega}=\left(\omega_{1}, \omega_{2}\right), \boldsymbol{d}=\left(d_{1}, d_{2}\right)$.

To shorten the notation, we define for all $\boldsymbol{\omega} \in \mathbb{R}^{s}$

$$
\Omega_{\boldsymbol{d}}(\boldsymbol{\omega}):=\frac{1-e^{-i\langle\boldsymbol{\lambda}, \boldsymbol{d}\rangle \omega}}{i \omega}
$$

Then $\widehat{\boldsymbol{B}}_{z}\left(\boldsymbol{\omega} \mid e ; \boldsymbol{d} \mathbb{N}_{0}\right)=\Omega_{\boldsymbol{d}}(\boldsymbol{\omega})^{z}$. For $\omega \geq 1$, we have

$$
\left|\widehat{\boldsymbol{B}}_{z}\left(\boldsymbol{\omega} \mid e ; \boldsymbol{d} \mathbb{N}_{0}\right)\right| \leq\left(\frac{2}{\omega}\right)^{\operatorname{Re} z} e^{-\operatorname{Im} z \arg \Omega_{\boldsymbol{d}}(\boldsymbol{\omega})}
$$

Thus $\left|\widehat{\boldsymbol{B}}_{z}\left(\boldsymbol{\omega} \mid e ; \boldsymbol{d} \mathbb{N}_{0}\right)\right| \in \mathcal{O}\left(\omega^{-\operatorname{Re} z}\right)$ for $\omega \rightarrow \infty$. Moreover, the decay depends on the phase of $\Omega_{\boldsymbol{d}}(\boldsymbol{\omega})$ and therefore on the direction $\boldsymbol{\lambda}$. The imaginary part $\operatorname{Im} z$ of the complex exponent allows for an enhancement of the corresponding frequency sectors. (Compare Figure 3 (b).)

The multivariate B-splines are discontinuous at the origin. The reason is that the radial limit behaves as

$$
\lim _{\omega \rightarrow 0} \widehat{\boldsymbol{B}}_{z}\left(\omega \boldsymbol{\lambda} \mid e, \boldsymbol{d} \mathbb{N}_{0}\right)=(\langle\boldsymbol{\lambda}, \boldsymbol{d}\rangle)^{z}
$$

i.e., the limit depends on the direction $\boldsymbol{\lambda}$. However, $\widehat{\boldsymbol{B}}_{z}$ is bounded on every neighborhood of the origin. In fact,

$$
\begin{aligned}
\left|\Omega_{\boldsymbol{d}}(\boldsymbol{\omega})\right| & =\left|\frac{1-e^{-i\langle\boldsymbol{\omega}, \boldsymbol{d}\rangle}}{i \omega}\right|=\left|\frac{-\sum_{k=1}^{\infty} \frac{1}{k !}(-i \omega\langle\boldsymbol{\lambda}, \boldsymbol{d}\rangle)^{k}}{i \omega}\right| \\
& \leq \frac{1}{\omega} \sum_{k=1}^{\infty} \frac{(\omega\|\boldsymbol{d}\|)^{k}}{k !}=\|\boldsymbol{d}\| \sum_{k=1}^{\infty} \frac{1}{k !} \omega^{k-1}\|\boldsymbol{d}\|^{k-1} \\
& \leq\|\boldsymbol{d}\| e^{\omega\|\boldsymbol{d}\|} .
\end{aligned}
$$

Hence for $|\omega| \leq 1$,

$$
\begin{aligned}
\left|\Omega_{\boldsymbol{d}}(\boldsymbol{\omega})^{z}\right| & \leq e^{\operatorname{Re} z \ln |\Omega(\boldsymbol{\omega})|} \cdot e^{-\operatorname{Im} z \arg \Omega(\boldsymbol{\omega})} \\
& \leq e^{\operatorname{Re} z \ln \|\boldsymbol{d}\|} e^{\operatorname{Re} z \omega\|\boldsymbol{d}\|} e^{-\operatorname{Im} z \arg \Omega(\boldsymbol{\omega})} \\
& \leq\|\boldsymbol{d}\|^{\operatorname{Re} z} e^{\operatorname{Re} z \omega\|d\|} e^{\pi \operatorname{Im} z} \\
& \leq\|\boldsymbol{d}\|^{\operatorname{Re} z} e^{\operatorname{Re} z\|d\|} e^{\pi \operatorname{Im} z}
\end{aligned}
$$

A representation of $\widehat{\boldsymbol{B}}_{z}$ in polar coordinates $(r, \theta)$ is shown in Figure 3.

As their 1D relatives, the cardinal B-splines, these multivariate B-splines 


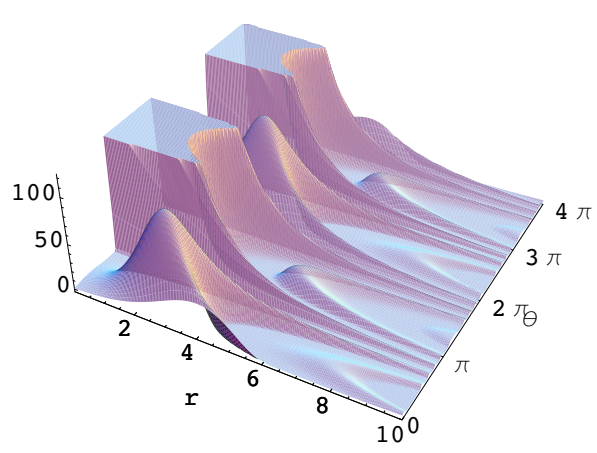

(a)

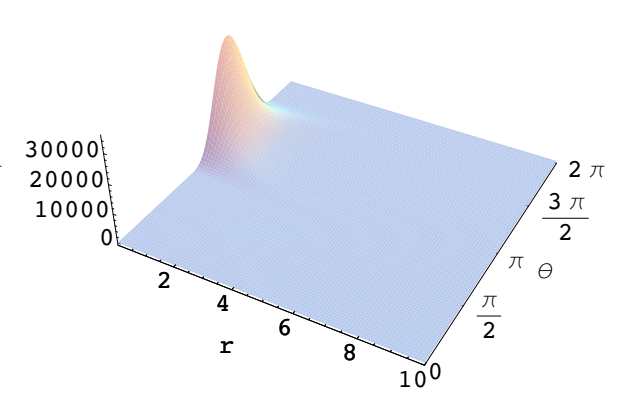

(b)

Figure 3: (a) Two periods of the complex multivariate B-spline in Fourier domain with respect to polar coordinates: $\widehat{\boldsymbol{B}}_{z}\left(r e^{i \theta} \mid e, \boldsymbol{d} \mathbb{N}_{0}\right)$ with $z=3+3 i$ and $d=$ $(1,1)^{T}$ as direction for the sequence of knots. (Large values are truncated.) (b) One period of the same spline with full range shown. The decay depends on the angle $\theta$.

satisfy a two-scale relation:

$$
\begin{aligned}
\frac{\widehat{\boldsymbol{B}}_{z}(2 \boldsymbol{\omega} \mid e, \boldsymbol{\tau})}{\widehat{\boldsymbol{B}}_{z}(\boldsymbol{\omega} \mid e, \boldsymbol{\tau})} & =\frac{\widehat{\boldsymbol{B}}_{z}\left(2 \boldsymbol{\omega} \mid e, \boldsymbol{d} \mathbb{N}_{0}\right)}{\widehat{\boldsymbol{B}}_{z}\left(\boldsymbol{\omega} \mid e, \boldsymbol{d} \mathbb{N}_{0}\right)} \\
& =\left(\frac{1-e^{-i\langle\boldsymbol{\lambda}, \boldsymbol{d}\rangle 2 \omega}}{2 i \omega}\right)^{z}\left(\frac{i \omega}{1-e^{-i\langle\boldsymbol{\lambda}, \boldsymbol{d}\rangle \omega}}\right)^{z} \\
& =\frac{1}{2^{z}}\left(1+e^{-i\langle\boldsymbol{\lambda}, \boldsymbol{d}\rangle \omega}\right)^{z} \\
& =\frac{1}{2^{z}}\left(1+e^{-i\langle\boldsymbol{\omega}, \boldsymbol{d}\rangle}\right)^{z}
\end{aligned}
$$

Here we used the equation

$$
\left(1+e^{-i\langle\boldsymbol{\lambda}, \boldsymbol{d}\rangle \omega}\right)\left(1-e^{-i\langle\boldsymbol{\lambda}, \boldsymbol{d}\rangle \omega}\right)=1-e^{-2 i\langle\boldsymbol{\lambda}, \boldsymbol{d}\rangle \omega} .
$$

The Fourier coefficients of the filter (7) are thus given by those of the generalized binomial formula:

$$
\frac{\widehat{\boldsymbol{B}}_{z}(2 \boldsymbol{\omega} \mid e, \boldsymbol{\tau})}{\widehat{\boldsymbol{B}}_{z}(\boldsymbol{\omega} \mid e, \boldsymbol{\tau})}=\frac{1}{2^{z}} \sum_{n=0}^{\infty}\left(\begin{array}{l}
z \\
n
\end{array}\right) e^{-i\langle\boldsymbol{\omega}, \boldsymbol{d}\rangle n} .
$$

Hence, the multivariate complex B-splines $\boldsymbol{B}_{z}(\bullet \mid e ; \boldsymbol{\tau})$ satisfy the two-scale or refinement equation

$$
\boldsymbol{B}_{z}(\bullet \mid e ; \boldsymbol{\tau})=\frac{1}{2^{z}} \sum_{n=0}^{\infty}\left(\begin{array}{l}
z \\
n
\end{array}\right) \boldsymbol{B}_{z}(2 \bullet-n \boldsymbol{d} \mid e ; \boldsymbol{\tau}) \quad \text { in } L^{2}\left(\mathbb{R}^{s}\right)
$$




\section{Time domain representation in the multivariate case}

For an explicit time domain representation, we consider the Fourier inversion of (5):

$$
\begin{aligned}
\boldsymbol{B}_{z}(\boldsymbol{x} \mid e, \boldsymbol{\tau}) & =\boldsymbol{B}_{z}\left(\boldsymbol{x} \mid e, \boldsymbol{d} \mathbb{N}_{0}\right) \\
& =\frac{1}{(2 \pi)^{s}} \int_{\mathbb{R}^{s}} \widehat{\boldsymbol{B}}_{z}\left(\boldsymbol{\omega} \mid e, \boldsymbol{d} \mathbb{N}_{0}\right) e^{i\langle\boldsymbol{x}, \boldsymbol{\omega}\rangle} d \boldsymbol{\omega} \\
& =\frac{1}{(2 \pi)^{s}} \int_{\mathbb{R}^{s}}\left(\frac{1-e^{-i\langle\boldsymbol{\omega}, \boldsymbol{d}\rangle}}{i\|\boldsymbol{\omega}\|}\right)^{z} e^{i\langle\boldsymbol{x}, \boldsymbol{\omega}\rangle} d \boldsymbol{\omega}
\end{aligned}
$$

In the frequency domain, the B-splines are products of a periodic function with a radial power function $1 /\|\boldsymbol{\omega}\|^{z}$. Therefore, in the time domain they can be represented as a semi-discrete convolution of the sequence of Fourier coefficients of

$$
\left(1-e^{-i\langle\boldsymbol{\omega}, \boldsymbol{d}\rangle}\right)^{z}=\sum_{n=0}^{\infty}\left(\begin{array}{l}
z \\
n
\end{array}\right)\left(-e^{-i\langle\boldsymbol{\omega}, \boldsymbol{d}\rangle}\right)^{n}=\sum_{n=0}^{\infty}(-1)^{n}\left(\begin{array}{l}
z \\
n
\end{array}\right) e^{-i n\langle\boldsymbol{\omega}, \boldsymbol{d}\rangle}
$$

with the Fourier inverse of the radial function $1 /\|\boldsymbol{\omega}\|^{z}$. The Fourier inverse of the Hadamard partie finie is given by [27, pp. $257 \mathrm{ff}$.

$$
\frac{1}{2^{\frac{z+s}{2}}} \pi^{\frac{z}{2}-s} \frac{\Gamma\left(\frac{s-z}{2}\right)}{\Gamma\left(\frac{z}{2}\right)} \mathcal{F}\left(r^{z-s}\right)=\operatorname{Pf}\left(r^{-z}\right)
$$

This yields the following result.

Proposition 11. The time domain representation of the multivariate complex B-spline is given by

$$
\boldsymbol{B}_{z}\left(\boldsymbol{x} \mid e, \boldsymbol{d} \mathbb{N}_{0}\right)=\frac{\pi^{\frac{z}{2}-s}}{i^{z} 2^{\frac{z+3 s}{2}}} \frac{\Gamma\left(\frac{s-z}{2}\right)}{\Gamma\left(\frac{z}{2}\right)} \sum_{n=0}^{\infty}(-1)^{n}\left(\begin{array}{l}
z \\
n
\end{array}\right)\|\boldsymbol{x}-n \boldsymbol{d}\|^{z-s},
$$

in the sense of tempered distributions and in $L^{2}\left(\mathbb{R}^{s}\right)$.

Note that, using Euler's reflection formula as well as the duplication formula for the Gamma function, the coefficient in front of the sum in (8) reduces to $1 / \Gamma(z)$ when $s=1$.

Figure 4 shows the B-splines $\boldsymbol{B}_{z}\left(\boldsymbol{x} \mid e, \boldsymbol{d} \mathbb{N}_{0}\right)$ in the space domain. Due to the decay (6) in the frequency domain, all partial derivatives of order $<\operatorname{Re} z-s$ are continuous in the space domain and are elements of $L^{2}\left(\mathbb{R}^{s}\right)$. 


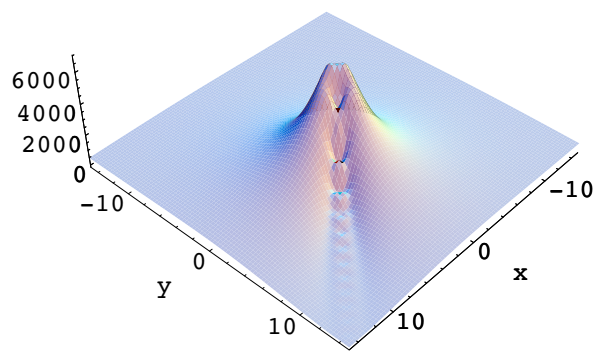

(a)

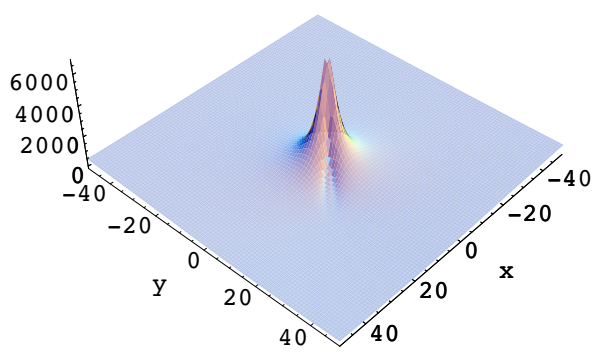

(c)

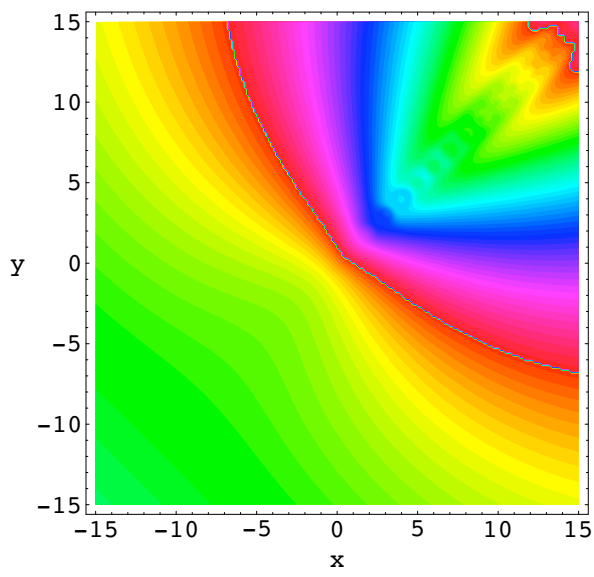

(b)

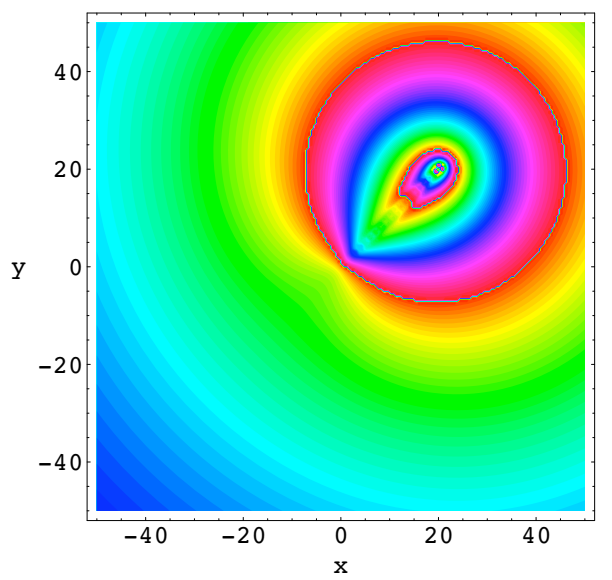

(d)

Figure 4: Space domain representation of the multivariate complex B-spline $\boldsymbol{B}_{z}\left(\boldsymbol{x} \mid e, \boldsymbol{d} \mathbb{N}_{0}\right)$ in $2 \mathrm{D}$ for the parameters $z=3+3 i$ and $\boldsymbol{d}=(1,1)^{T}$ in a neighborhood of the origin (a,b), and on a larger scale (c,d). Left column: Absolute values. Right column: Phase. Note the knots on the ray $x=y$. The function is continuous for this choice of $z$. 


\section{Fractional Differential Operators and Splines of Com- plex Order}

In this section, we define a new class of splines, namely, splines of complex order $z, \operatorname{Re} z>1$, and show that the complex B-splines introduced earlier are examples of such splines. These new splines are distributional solutions of a certain fractional differential equation and extend the ideas and concepts presented, for instance, in $[13,30]$.

In order to present this new class of splines of complex order, we need to introduce the following two subspaces of the Schwartz space $\mathcal{S}:=\mathcal{S}\left(\mathbb{R}^{n}\right)$. These spaces play an important role in the theory of fractional differentiation and integration as they allow the identification of several fractional differential and integral operators.

To this end, denote by $\Delta: \mathcal{S} \rightarrow \mathcal{S}, \Delta:=-\sum_{j=1}^{n} \partial_{j}^{2}$, the Laplace operator on $\mathcal{S}$. Here, $\partial_{j}$ denotes the partial derivative with respect to the $j$-th coordinate of $\mathbb{R}^{n}$. Then, the Lizorkin spaces $\Phi$ and $\Psi$ are defined by

$$
\Phi:=\bigcap_{m \in \mathbb{N}} \Delta^{m}(\mathcal{S})
$$

i.e., $\Phi$ is the space of all Schwartz functions that are orthogonal to all polynomials, and

$$
\Psi:=\left\{\psi \in \mathcal{S}: \partial^{\mu} \psi(\mathbf{0})=0, \forall \mu \in \mathbb{N}^{n}\right\},
$$

respectively. Here, $\mu$ is a multi-index of length $n$ and $\partial^{\mu}$ describes the partial differentiation with respect to the canonical coordinates of $\mathbb{R}^{n}$.

The next proposition characterizes the elements of $\Psi$.

Proposition 12. For all $\psi \in \mathcal{S}$ are equivalent:

(i) $\psi \in \Psi$;

(ii) $\left(\partial^{\mu} \psi\right)(\boldsymbol{\xi}) \in o\left(\|\boldsymbol{\xi}\|^{t}\right)$ as $\|\boldsymbol{\xi}\| \rightarrow 0, \forall \mu \in \mathbb{N}^{n}, \forall t \in \mathbb{R}^{+}$;

(iii) $\|\boldsymbol{\xi}\|^{-2 m} \psi \in \mathcal{S}, \forall m \in \mathbb{N}$.

Proof. See, for instance, [28].

Using Proposition 12, one can show the next result.

Proposition 13. The following relationship holds between $\Phi$ and $\Psi$, namely, $\mathcal{F}(\Phi)=\Psi$, where $\mathcal{F}$ denotes the Fourier transform.

Proposition 14. Let $\mathcal{S}^{\prime}:=\mathcal{S}^{\prime}\left(\mathbb{R}^{n}\right)$ be the topological dual of $\mathcal{S}$. Then the topological duals of $\Phi$ and $\Psi$ are as follows.

(i) $\Phi^{\prime}=\mathcal{S}^{\prime} / \mathcal{P}$. 
(ii) $\Psi^{\prime}=\mathcal{S}^{\prime} / \mathcal{F}(\mathcal{P})$,

where, $\mathcal{P}:=\left\{f \in \mathcal{S}^{\prime}: \operatorname{supp} \mathcal{F}(f)=\{0\}\right\}$ denotes the set of polynomials in $\mathcal{S}^{\prime}$.

For the proofs of these proposition, we refer the reader to [26] or [28].

Regarding the spaces $\mathcal{P}$ and $\Psi$, we note that $f \in \mathcal{P}$ if and only if $\Delta^{m} f=0$, for some $m \in \mathbb{N}$, and that $\Psi$ is dense in $L^{p}\left(\mathbb{R}^{n}\right)$ for $1 \leq p<\infty$. (Cf. [28].)

For the remainder of this section, we now suppose that $n:=1$.

For our purposes, we need the restriction of $\Psi$ to the non-negative half-axis:

$$
\Psi_{+}:=\{f \in \Psi: \operatorname{supp} f \subseteq[0, \infty)\}
$$

Note that the properties mentioned in Propositions 12 - 14 easily carry over to $\Psi_{+}$.

Let $f \in \Psi_{+}$and let $k_{z}: \mathbb{R} \rightarrow \mathbb{C}, x \mapsto \frac{x_{+}^{z-1}}{\Gamma(z)}$, with $\operatorname{Re} z>0$.

Definition 15. Suppose that $z \in \mathbb{C}_{+}:=\{\zeta: \operatorname{Re} \zeta>0\}$. We define a fractional derivative operator $\mathcal{D}^{z}$ of complex order $z$ on $\Psi_{+}$by

$$
\mathcal{D}^{z} f:=\left(D^{n} f\right) * k_{n-z}=D^{n}\left(f * k_{n-z}\right), \quad n=\lceil\operatorname{Re} z\rceil,
$$

where $*$ denotes the convolution on $\Psi_{+}$and $D^{n}$ the derivative in the distributional sense.

Similarly, a fractional integral operator $\mathcal{D}^{-z}$ of complex order $z$ on $\Psi_{+}$is defined by

$$
\mathcal{D}^{-z} f:=f * k_{z}
$$

Remark 16. If we consider function spaces other than $\Psi_{+}$, then $\left(D^{n} f\right) * k_{n-z} \neq$ $D^{n}\left(f * k_{n-z}\right)$ and each side defines a different fractional derivative operator. For more details about these different fractional derivatives, which go by the names of Caputo and Riemann-Liouville, the interested reader may consult [15] or [25]

It follows easily from the convolution-based definition of fractional derivative, respectively, integral, that

$$
f \in \Psi_{+} \quad \Longrightarrow \quad \mathcal{D}^{ \pm z} f \in \Psi_{+} .
$$

Since $\mathcal{S}$ is invariant under $\mathcal{D}^{ \pm z}$, it remains to be shown that all derivatives of $\mathcal{D}^{ \pm z} f$ vanish at $x=0$. However, for all $m \in \mathbb{N}_{0}$ we have

$$
D^{m}\left(f * k_{z}\right)(0)=\left.\left(D^{m} f\right) * k_{z}\right|_{x=0}=\left.\frac{1}{\Gamma(z)} \int_{0}^{x}\left(D^{m} f\right)(t)(x-t)^{z-1} d t\right|_{x=0}=0 .
$$

Using a similar argument, the above equality also shows that $\mathcal{D}^{z} f \in \Psi_{+}$. 
The convolution-based definition of the fractional derivative and integral of functions $f \in \Psi_{+}$also implies that $\left\{\mathcal{D}^{z}: z \in \mathbb{C}\right\}$ is a semi-group in the sense that

$$
\mathcal{D}^{z+\zeta}=\mathcal{D}^{z} \mathcal{D}^{\zeta}=\mathcal{D}^{\zeta} \mathcal{D}^{z}=\mathcal{D}^{\zeta+z}
$$

for all $z, \zeta \in \mathbb{C}$. (For a proof, see [25], pp. 83 - 84.)

Next, we define the fractional derivative/integral operator $\mathcal{D}^{ \pm z}$ on the dual space $\Psi_{+}^{\prime}$ of $\Psi_{+}$. To this end, we may regard the locally integrable function $k_{z}$, $\operatorname{Re} z>-1$, as an element of $\Psi_{+}^{\prime}$ by setting

$$
\left\langle k_{z}, \varphi\right\rangle=\int_{0}^{\infty} k_{z}(t) \varphi(t) d t, \quad \forall \varphi \in \Psi_{+} .
$$

Here, $\langle\bullet, \bullet\rangle$ denotes the canonical pairing between $\Psi_{+}$and $\Psi_{+}^{\prime}$. In passing, we note that the function $z \mapsto\left\langle k_{z}, \varphi\right\rangle, \forall \varphi \in \Psi_{+}$, is holomorphic for all $z \in \mathbb{C} \backslash \mathbb{N}_{0}$.

Remark 17 . The function $k_{z}$ may also be defined for general $z \in \mathbb{C}$ via Hadamard's partie finie and represents then a pseudo-function. For more details, we refer the interested reader to $[7,12]$, or [31].

Note that for $f, g \in \Psi_{+}^{\prime}$ the convolution exists on $\Psi_{+}^{\prime}$ and is defined in the usual way (see, for instance, [26]) by

$$
\langle f * g, \varphi\rangle:=\langle(f \times g)(x, y), \varphi(x+y)\rangle=\langle f(x),\langle g(y), \varphi(x+y)\rangle\rangle, \quad \varphi \in \Psi_{+} .
$$

The pair $\left(\Psi_{+}^{\prime}, *\right)$ is a convolution algebra with the Dirac delta distribution $\delta$ as its unit element.

Definition 18. Let $z \in \mathbb{C}_{+}$, let $\varphi \in \Psi_{+}$be a test function and $f \in \Psi_{+}^{\prime}$. Then the fractional derivative operator $\mathcal{D}^{z}$ on $\Psi_{+}^{\prime}$ is defined by

$$
\left\langle\mathcal{D}^{z} f, \varphi\right\rangle:=\left\langle\left(D^{n} f\right) * k_{n-z}, \varphi\right\rangle, \quad n=\lceil\operatorname{Re} z\rceil,
$$

and the fractional integral operator $\mathcal{D}^{-z}$ by

$$
\left\langle\mathcal{D}^{-z} f, \varphi\right\rangle:=\left\langle f * k_{z}, \varphi\right\rangle .
$$

The semi-group properties (9) also hold for $f \in \Psi_{+}^{\prime}$. (For a direct proof, see [12] or [25].)

Example 19. Let $z \in \mathbb{C}_{+}$. Then it can be shown (cf. $[12,25,26]$ ) that the $z$-th derivative of a truncated power function is given

$$
\mathcal{D}^{z}\left[\frac{(x-k)^{z-1}}{\Gamma(z)}\right]=\delta(x-k), \quad k<x \in[0, \infty) .
$$

Thus, employing the semi-group properties of $\mathcal{D}^{z}$ or by direct computation using the definition (10) of convolution, one obtains

$$
\mathcal{D}^{-z} \delta(\bullet-k)=\frac{(\bullet-k)_{+}^{z-1}}{\Gamma(z)} .
$$


Based on the original ideas in $[13,30]$ to define splines via certain classes of differential operators and the description in [17], we introduce splines of complex order $z, z \in \mathbb{C}_{+}$as follows.

Definition 20. Let $z \in \mathbb{C}_{+}$and let $\left\{a_{k}: k \in \mathbb{N}_{0}\right\} \in \ell^{\infty}(\mathbb{R})$. A solution of the equation

$$
\mathcal{D}^{z} f=\sum_{k=0}^{\infty} a_{k} \delta(\bullet-k)
$$

is called a spline of complex order $z$.

Remarks 21. (i) If in (13) we set $z:=n \in \mathbb{N}$, then we obtain the cardinal B-spline of order $n$ as a solution. (See, for instance, $[13,17,30]$.)

(ii) The right-hand side of (13) may be interpreted as a weighted Dirac comb or $\amalg$-function.

To prove the existence of splines of complex order $z \in \mathbb{C}_{+}$, we show that the complex B-splines first introduced in [3] and later explored and generalized in $[9,11,18,16]$ provide a solution to Equation (13).

Proposition 22. The complex B-spline

$$
B_{z}(x)=\frac{1}{\Gamma(z)} \sum_{k=0}^{\infty}(-1)^{k}\left(\begin{array}{l}
z \\
k
\end{array}\right)(x-k)_{+}^{z-1}, \quad \operatorname{Re} z \geq 1 .
$$

is a solution of Equation (13).

Proof. Let $z \in \mathbb{C}$ with $\operatorname{Re} z \geq 1$. By the linearity of $\mathcal{D}^{z}$ and Equation (11) we obtain

$$
\mathcal{D}^{z} B_{z}(x)=\sum_{k=0}^{\infty}(-1)^{k}\left(\begin{array}{l}
z \\
k
\end{array}\right) \mathcal{D}^{z}\left[\frac{(x-k)_{+}^{z-1}}{\Gamma(z)}\right]=\sum_{k=0}^{\infty}(-1)^{k}\left(\begin{array}{l}
z \\
k
\end{array}\right) \delta(x-k) .
$$

We note that the infinite series $\sum_{k=0}^{\infty}(-1)^{k}\left(\begin{array}{l}z \\ k\end{array}\right)$ is bounded above by $c e^{|z-1|}, c>0$. (See [3] for a verification.)

The afore-mentioned approach can be extended to a class of linear differential operators of complex order with constant coefficients of the form

$$
\mathcal{L}^{z}:=\prod_{i=1}^{n}\left(\mathcal{D}^{z_{i}}-\lambda_{i} I\right)
$$

where $z=\left(z_{1}, \ldots, z_{n}\right) \in \mathbb{C}_{+}^{n}$ with $0<\operatorname{Re} z_{i} \leq 1, \lambda_{i} \in \mathbb{R}$ with $\lambda_{i} \neq \lambda_{j}$ for $i \neq j ; i, j=1, \ldots, n$, and $n \in \mathbb{N}$. Here, $I$ denotes the identity operator. Since 
the fractional derivative operator satisfies the semi-group properties (9), the solution to the equation

$$
\mathcal{L}^{z} f=0
$$

where $f \in \Psi_{+}^{\prime}$, is given by

$$
f=\sum_{i=1}^{n} c_{i} E_{z_{i}}\left(\lambda_{i}(\bullet)^{z_{i}}\right), \quad c_{i} \in \mathbb{R} .
$$

In Equation (14), the Mittag-Leffler function $E_{z}$ is given by

$$
E_{z}(x):=\sum_{k=0}^{\infty} \frac{x^{k}}{\Gamma(k z+1)}, \quad z \in \mathbb{C}_{+} .
$$

Equation (14) provides the starting point for the definition of more general splines of complex order $z$ and the construction of associated local bases of Bspline type. Here, however, we will not pursue this topic further but refer the interested reader to an upcoming paper on this new subject.

\section{Summary}

Complex B-splines allow the definition of difference and divided difference operators of complex order for arbitrary knots and weights. Via their relation to Dirichlet averages and Dirichlet splines, they can be extended to higher dimensions by employing ridge functions. The Fourier transforms of the univariate and multivariate complex B-splines are also related via the projection of knots onto the ridge.

We gave explicit representations of complex B-splines in space and frequency domain. The multivariate versions of the complex B-spline are also represented by a series of shifted polynomials, and therefore the term 'spline' in this context is justified. The real part of the complex exponent yields the order of decay in the frequency domain and thus the regularity in the space domain. Finally, we considered univariate splines of complex order as solutions of certain linear fractional differential operators and a Dirac comb series.

\section{References}

[1] H. B. Curry and I. J. Schoenberg, On spline distributions and their limits: The Pólya distribution functions, Bulletin of the AMS, 53, no. 7-12, p. 1114, 1947, abstract.

[2] C. de Boor, Splines as linear combinations of B-splines, in Approximation Theory II, G. G. L. et al., Ed. Academic Press, 1976, pp. 1-47. 
[3] B. Forster, T. Blu, and M. Unser, Complex B-splines, Appl. Comp. Harmon. Anal., 20, pp. 281-282, 2006.

[4] B. C. Carlson, Special Functions of Applied Mathematics. Academic Press, New York, 1977.

[5] — B-Splines, hypergeometric functions and Dirichlet averages, J. Approx. Th., 67, 311-325, 1991.

[6] W. Dahmen and C. A. Micchelli, Statistical Encounters with B-Splines, Contemporary Mathematics, 59, 17-48, 1986.

[7] R. Dantray and J.-L. Lions, Mathematical Analysis and Numerical Methods for Science and Technology, Vol. 2. Springer Verlag, Berlin, Germany, 2000 .

[8] C. L. Epstein, Mathematics of Medical Imaging. Prentice-Hall, 2003.

[9] B. Forster and P. Massopust, Statistical encounters with complex B-Splines, Constructive Approximation, 29(3), . 325-344, 2009.

[10] - Some remarks about the connection between fractional divided differences, fractional B-Splines, and the Hermite-Genocchi formula, International Journal of Wavelets, Multiresolution and Information Processing, 6(2), 279-290, 2008.

[11] — Multivariate complex B-splines, Dirichlet averages and difference operators, Proceedings of SampTA, 2009.

[12] I. Gel'fand and G. Shilov, Generalized Functions, Vol. 1 (in Russian). Nauka, Moscow, Russia, 1959.

[13] S. Karlin, C. Micchelli, A. Pinkus, and I. Schoenberg, Studies in Spline Functions and Approximation Theory. Academic Press, New York, 1976.

[14] S. Karlin, C. A. Micchelli, and Y. Rinott, Multivariate splines: A probabilistic perspective, Journal of Multivariate Analysis, 20, 69-90, 1986.

[15] A. A. Kilbas, H. M. Srivastava, and J. J. Trujillo, Theory and Applications of Fractional Differential Equations. Elsevier B. V., Amsterdam, The Netherlands, 2006.

[16] P. Massopust, Double Dirichlet averages and complex B-splines, in Proceedings of SampTA, 2009.

[17] - Interpolation and Approximation with Splines and Fractals. Oxford University Press, New York, 2010. 
[18] P. Massopust and B. Forster, Multivariate complex B-splines and Dirichlet averages, Journal of Approximation Theory, 162, 252-269, 2010.

[19] C. A. Micchelli, A constructive approach to Kergin interpolation in $\mathbb{R}^{k}$ : Multivariate B-splines and Lagrange interpolation, Rocky Mt. J. Math., 10(3), 485-497, 1980.

[20] K. S. Miller and B. Ross, An introduction to the fractional calculus and fractional differential equations. Wiley, 1993.

[21] F. Natterer and F. Wübbeling, Mathematical Methods on Image Reconstruction, ser. Monographs on Mathematical Modeling and Computation. SIAM, 2001.

[22] E. Neuman and P. J. Van Fleet, Moments of Dirichlet splines and their applications to hypergeometric functions, Journal of Computational and Applied Mathematics, 53, 225-241, 1994.

[23] O. V. Odinokov, Spectral analysis in certain spaces of entire functions of exponential type and its applications, Izv. Math., 64(4), 777-786, 2000.

[24] A. Pinkus, Approximating by ridge functions, in Surface Fitting and Multiresolution Methods, A. Le Méhauté, C. Rabut, and L. L. Schumaker, Eds. Vanderbilt University Press, 1997, pp. 1-14.

[25] I. Podlubny, Fractional Differential Equations. Academic Press, 1999.

[26] S. G. Samko, A. A. Kilbas, and O. I. Marichev, Fractional Integrals and Derivatives. Gordon and Breach Science Publishers, Minsk, Belarus, 1987.

[27] L. Schwartz, Théorie des distributions. Hermann, 1998, reprint. Original of 1966 .

[28] M. Troyanov, On the Hodge decomposition in $\mathbb{R}^{n}$, Mosc. Math. J., 9(4), 899-926, 2009.

[29] M. Unser and T. Blu, Fractional splines and wavelets, SIAM Review, 42(1), 43-67, March 2000.

[30] - Cardinal exponential splines: Part i - theory and filtering algorithms, IEEE Trans. Signal Processing, 53(4), 1425-1438, 2005.

[31] A. H. Zemanian, Distribution Theory and Transform Analysis - An Introduction to Generalized Functions, with Applications. Dover Publications, Inc., New York, 1987.

[32] W. zu Castell, Dirichlet splines as fractional integrals of B-splines, Rocky Mt. J. Math., 32, 545-559, 2002. 